\title{
The influence of the non-linear diffusion on the mass transfer of a packaging contaminant into foodstuffs
}

\author{
Gheorghe Juncu
}

\begin{abstract}
The unsteady mass transfer of the packaging constituents to a food has been analysed. The diffusion coefficient inside the packaging was considered concentration - variable while the food was considered concentration spatially gradientless. A well-known technique (Kirchhoff transformation) was used to solve the non-linear mass balance equation. The influence of the non-linear diffusion on the mass transfer mechanism and rate was analysed for different values of the partition coefficient and dilution coefficient.
\end{abstract}

\section{Introduction}

The packaging materials used for food products contain different constituents (additives, monomers, antioxidants, etc.) known to be carcinogenetic. These constituents diffuse into the food products during processing, storage and transportation. In Europe, all materials intended to be in contact with foods must comply with the framework regulation 2004/1935/EC. As a result, the mathematical modeling of contaminants migration from packaging to food received an increased attention during the last years. Also, the EU regulation on the safety of chemicals encourages the development of mathematical models

Key Words: mass transfer; packaging; contaminants; food safety; concentration-variable diffusivity; Kirchhoff transformation.

2010 Mathematics Subject Classification: 65F10, 65F20.

Received: December, 2016.

Revised: March, 2017.

Accepted: August, 2017 
to assess the migration of chemicals into the environment.

The mathematical models widely used to describe this migration phenomenon consider that the diffusion inside the packaging controls the process (Begley et al. [1], Brandsch et. al. [2], Brandsch et. al. [3], Vitrac \& Hayert [10], Vitrac et al. [11]). The migration of the contaminants inside the packaging is described by the 1D Fick's second law with constant diffusion coefficients. The transport of the contaminants into the food, at the interface between the packaging and the food, is modeled by a Robin boundary condition. The other boundary of the packaging is considered an impervious boundary or a symmetric plane boundary condition. Pigeonneau et al. [9] recently proposed a conjugate mass transfer model that uses the Quasi Steady State Approximation for the transport of the contaminants into the food.

The packaging materials are polymers. The diffusion coefficients in polymers depend on the concentration of the diffusion species (Neogi [8], Hedenqvist \& Gedde [5]). This phenomenon, i.e. non-linear diffusion, was not analysed until now in migration analysis.

The investigation of the influence of the non-linear diffusion on the mass transfer of a contaminant from a packaging into a food is the aim of the present work. The mathematical model used is that developed by Vitrac \& Hayert [10] (see also Vitrac et al. [11]). The computations focus on the influence of the non-linear diffusion on the mass transfer rate for different values of the partition coefficient and dilution coefficient. This problem can be also viewed as one of interest for solar energy devices, (Mittelman et al. [7]).

This work is organized as follows. In Sect. 2 we describe the mathematical model of the problem. Section 3 presents the numerical algorithm. The numerical experiments made and the results obtained are presented in Sect. 4. Finally, some concluding remarks are briefly mentioned in Sect. 5 .

\section{Model Equations}

The transport of the contaminant in the polymer matrix is governed by the process of molecular diffusion. The food is considered a liquid (assumption recommended by the EU regulations). The liquid in contact is non-interacting with the polymer (no swelling, no plasticization). As a result, the dimensionless concentration of contaminant $Z=C_{P} / C_{P, 0}\left(C_{P}\right.$ is the instantaneous mass concentration of the migration species inside the packaging and $C_{P, 0}$ is the initial mass concentration of the migration species inside the packaging) is given by the dimensionless transport equation: 


$$
\frac{\partial Z}{\partial \tau}=\frac{\partial}{\partial X}\left[D(Z) \frac{\partial Z}{\partial X}\right]
$$

where $\tau$ is the dimensionless time or Fourier number and $X$ is the dimensionless spatial coordinate. The dimensionless variables are defined by:

$$
X=\frac{x}{L_{P}}, \tau=\frac{t D_{P, 0}}{L_{P}^{2}},
$$

where $D_{P, 0}$ is the diffusion coefficients of the migration species in the packaging when $C_{P}=C_{P, 0}, t$ is the time, $x$ is the Cartesian coordinate of the main transport direction and $L_{P}$ is the characteristic length scale of the packaging (see figure 1).

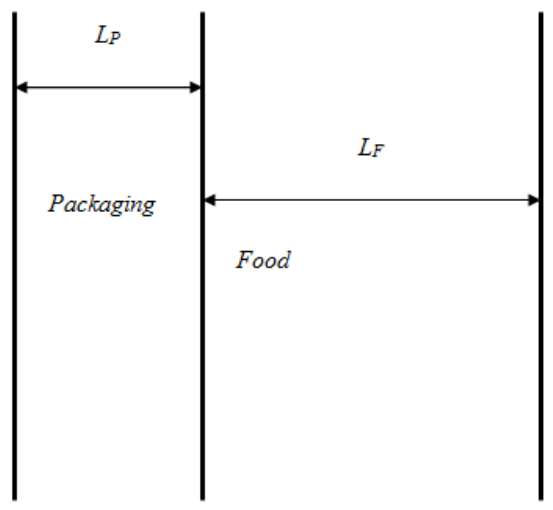

Figure 1: Schematic of the problem

Considering valid for the diffusion in the packaging the free-volume theory, (Fujita [4]), the dimensional diffusion coefficient $D_{P}\left(C_{P}\right)$ is given by:

$$
D_{P}\left(C_{P}\right)=D_{P, 0} \exp \left[-\beta\left(1-\frac{C_{P}}{C_{P, 0}}\right)\right]
$$

where $\beta$ is a dimensionless constant. The dimensionless diffusion coefficient $D(Z)=D_{P}\left(C_{P}\right) / D_{P, 0}$ is given by:

$$
D(Z)=\exp [-\beta(1-Z)]
$$


The boundary conditions are:

- axis of symmetry or impervious boundary, $X=0$;

$$
\frac{\partial Z}{\partial X}=0
$$

- interface between packaging and food, $X=1$;

$$
J=-\left.D(Z) \frac{\partial Z}{\partial X}\right|_{X=1}=\frac{B i}{K}\left(Z-Z_{0}^{\infty}\right)-B i \lambda \int_{0}^{\tau} J \mathrm{~d} \tau
$$

In relation (5), $B i=h_{F} L_{P} / D_{P, 0}$ is a dimensionless number that compares the values of transport resistances between packaging and food, $h_{F}$ is the equivalence conductance of the food, $K$ is the partition coefficient defined as (packaging concentration) / (food concentration), $Z_{0}^{\infty}$ is the initial concentration of the contaminant in the food and $\lambda=\left(\rho_{P} L_{P}\right) /\left(\rho_{F} / L_{F}\right)$ is the dilution coefficient with $\rho_{F}, \rho_{P}$ the densities of the food and packaging, respectively. The derivation of the boundary condition (5) (a generalized Robin boundary condition) can be viewed in Vitrac \& Hayert [10] and Vitrac et al. [11]. The dimensionless initial condition is:

$$
\tau=0, Z=1
$$

One of the physical quantities of interest for the present problem is the instantaneous dimensionless average concentrations in the packaging, $\bar{Z}$. The instantaneous dimensionless average concentration in the packaging was calculated with the relation:

$$
\bar{Z}=\int_{0}^{1} Z \mathrm{~d} X
$$

\section{Method of Solution}

The present mathematical model is a non-linear diffusion equation. An elegant method for solving non-linear diffusion equations is the use of the Kirchhoff transformation [6],

$$
\zeta=\int_{1}^{Z} D(Z) \mathrm{d} Z
$$

Applying the Kirchhoffs integral transformation (8) to the problem (1), (5), and taking into account the relations (Leibniz rule for differentiation) : 


$$
\frac{\partial \zeta}{\partial X}=D(Z) \frac{\partial Z}{\partial X}, \frac{\partial \zeta}{\partial \tau}=D(Z) \frac{\partial Z}{\partial \tau}
$$

the mathematical model becomes,

$$
\frac{\partial \zeta}{\partial \tau}=D(Z) \frac{\partial^{2} \zeta}{\partial X^{2}}
$$

with the boundary conditions:

- axis of symmetry or impervious boundary, $X=0$;

$$
\frac{\partial \zeta}{\partial X}=0
$$

- interface between packaging and food, $X=1$;

$$
J=-\left.\frac{\partial \zeta}{\partial X}\right|_{X=1}=\frac{B i}{K}\left(Z-Z_{0}^{\infty}\right)-B i \lambda \int_{0}^{\tau} J \mathrm{~d} \tau,
$$

The initial condition is:

$$
\tau=0, \zeta=0
$$

The relation between $Z$ and $\zeta$ is:

$$
Z=1+\frac{\ln (\beta \zeta+1)}{\beta}
$$

The mass balance equation (9) was solved numerically. The spatial derivatives were discretized with the central, second order accurate finite difference scheme. The flux boundary conditions (10), (11) were also discretized with the central second - order accurate scheme considering that the solution can be symmetrically extrapolated with one grid point outside the boundary. Numerical experiments were made on grids with the discretization step $\Delta X=1 / 64,1 / 128$ and $1 / 256$. The result of the spatial discretization is a system of ODEs. The MATLAB solvers ode15s and ode23tb were used to solve this system of ODEs.

For $\beta=0$, equation (1) with boundary conditions (4), (5) were solved numerically using the method described previously.

\section{Results}

The parameters of the present mathematical model are: $B i, K, Z_{0}^{\infty}, \beta$ and $\lambda$. According to Vitrac \& Hayert [10], the values considered in this work for $B i$ 
are: $B i=1,10$ and 100 . The partition coefficient $K$ takes values in the range $1-10^{3}$ (according to EU directives, Brandsch et al. [3]). The values considered for the dilution ratio are $\lambda=10^{-1}, 10^{-2}$, and $10^{-3}$. The dimensionless diffusion constant $\beta$ takes values from 0 to 10 . In all numerical simulations $Z_{0}^{\infty}$ was considered equal to, $Z_{0}^{\infty}=0$.

Results that can be used to validate the accuracy of the present computations are not available in literature. However, the present problem has a steady state solution with constant dimensionless concentrations in both phases (thermodynamic equilibrium). This dimensionless, steady state, thermodynamic equilibrium solution, $Z_{e c h}$, is given by:

$$
Z_{\text {ech }}=\frac{K}{K+\frac{1}{\lambda}}
$$

The agreement between the steady state solutions obtained numerically and those calculated with relation (14) was excellent. Relation (14) is given by the overall mass balance of the space averaged contaminant concentration when $\tau \rightarrow \infty,[10]$.

The effect of the non-linear diffusion parameter $\beta$ on the mass transfer (diffusion) rate depends on the contaminant concentration values (see relation (3)). The concentration of the contaminant inside the packaging varies in time from the initial value (relation (6)) to the thermodynamic equilibrium value (relation (14)). Therefore, the thermodynamic equilibrium value of the contaminant concentration plays a key role in the present analysis. A negligible effect of $\beta$ is expected for high values of $Z_{\text {ech }}$. The effect of $\beta$ should increase for small values of $Z_{\text {ech }}$.

Figures $2-4$ confirm these assumptions.

When $Z_{\text {ech }} \approx 0.9$ or greater than $0.9, \beta$ does not influence the mass transfer rate (figure 2 ). The decrease in $Z_{e c h}$ increases significantly the effect of $\beta$ on the mass transfer rate (figures 3 and 4 ).

The effect of $B i$ on the mass transfer rate depends on the values of $\beta$ and $Z_{\text {ech }}$. For $\beta=0$, the increase in $B i$ increases the mass transfer rate regardless the values of $Z_{\text {ech }}$ (see figures 5 and 6 ). If $\beta>0$, the influence of $B i$ on the mass transfer rate depends on the values of $Z_{e c h}$. For high values of $Z_{e c h}$ the effect of $B i$ remains the same as in the case $\beta=0$. The decrease in $Z_{\text {ech }}$ decreases the effect of $B i$. For small values of $Z_{\text {ech }}$, the effect of $B i$ on the mass transfer rate becomes negligible. 


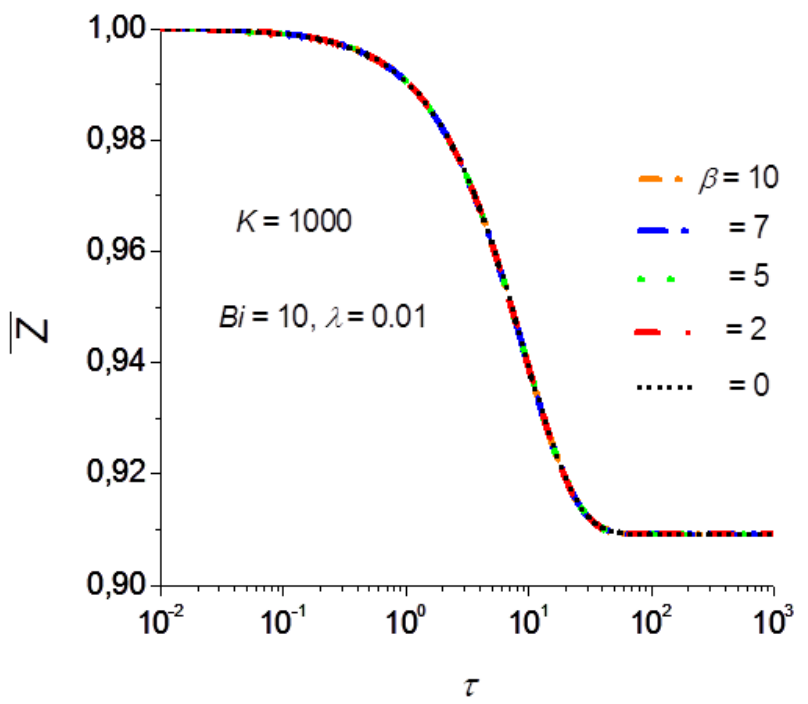

Figure 2: Time variation of the average dimensionless concentration for $B_{i}=$ 10 and $Z_{\text {ech }}=0.909$

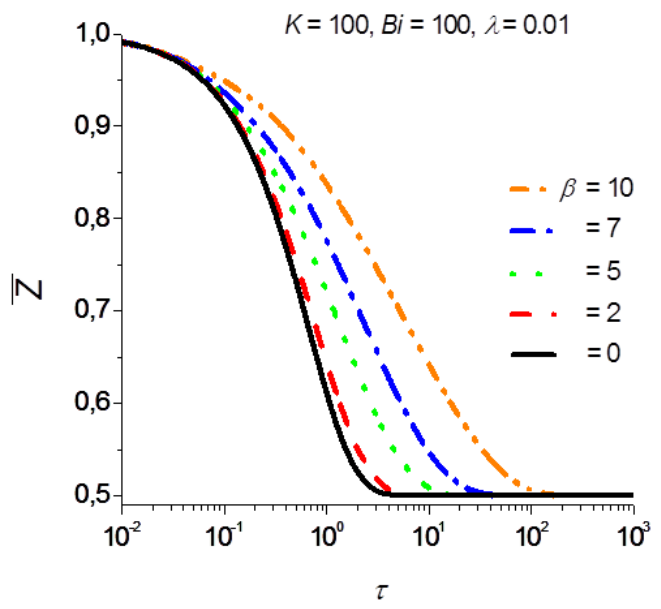

Figure 3: Time variation of the average dimensionless concentration for $B_{i}=$ 100 and $Z_{\text {ech }}=0.50$ 


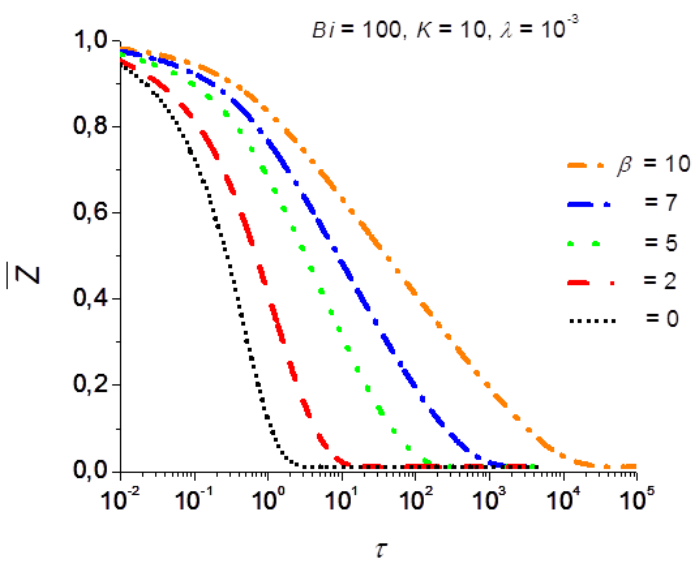

Figure 4: Time variation of the average dimensionless concentration for $B_{i}=$ 100 and $Z_{\text {ech }}=0.0099$
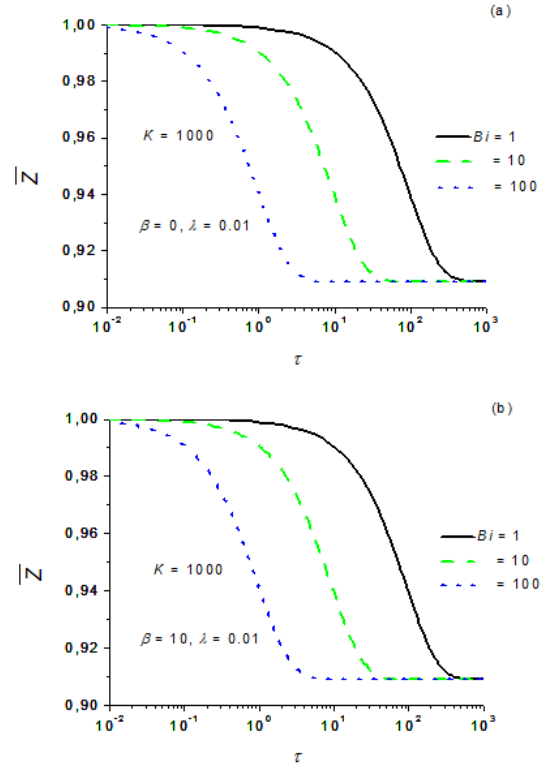

Figure 5: The effect of $B_{i}$ on time variation of the average dimensionless concentration; $Z_{\text {ech }}=0.909$; (a) $\beta=0$; (b) $\beta=10$ 

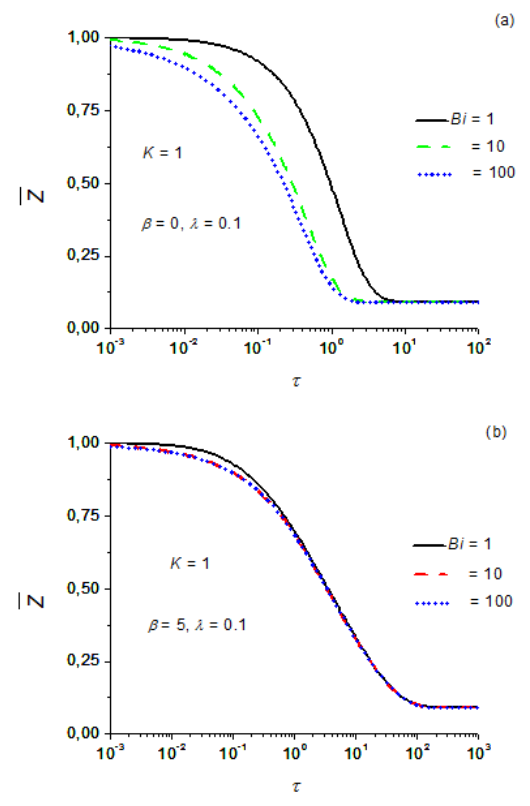

Figure 6: The effect of $B_{i}$ on time variation of the average dimensionless concentration; $Z_{e} c h=0.0909$; (a) $\beta=0$; (b) $\beta=5$

\section{Conclusions}

This work investigated numerically the influence of the non-linear diffusion on the unsteady mass transfer of a contaminant from a packaging to a food. The free volume theory (Fujita model) was employed for the non-linear diffusion in the packaging. The numerical results presented in the previous section can be summarized as follows:

- the non-linear dimensionless parameter $\beta$ influences the mass transfer rate only for moderate and small values of the thermodynamic equilibrium dimensionless concentration, $Z_{e c h}$; it increases the time necessary to reach the steady state, thermodynamic equilibrium solution;

- the influence of the dimensionless number that compares the values of transport resistances between packaging and food, $B i$, on the mass transfer rate decreases with the decrease in $Z_{\text {ech }}$;

- the values of $Z_{\text {ech }}$ are given by the values of the partition coefficient $K$ and the dilution ratio $\lambda$. 


\section{References}

[1] T. Begley, L. Castle, A. Feigenbaum, R. Franz, K. Hinrichs, T. Lickly, P. Mercea, M. Milana, A. OBrien, S. Rebre, R. Rijk, O. Piringer, Evaluation of migration models that might be used in support of regulations for food contact plastics, Food Additives \& Contaminants , 22 (2005), 73-90.

[2] J. Brandsch, P. Mercea, M. Rueter, V. Tosa, O. Piringer, Migration modeling as a tool for quality assurance of food packaging, Food Additives \& Contaminants , 19Suppl (2002), 29-41.

[3] R. Brandsch, B. Brands, R. Franz, M. Klatt, M.R. Milana, O. Piringer, A. Schaefer, C. Simoneau, X. Trier, O. Virac, Applicability of generally recognized diffusion models for the estimation of specific migration in support of EU Directive 2002/72/EC, In C. Simoneau, editor, JRC Scientific and Technical Reports, number EUR 24514 EN 2010, Publications Office of the European Union (2010).

[4] H. Fujita, Diffusion in polymer diluent systems, Algebra Universalis , 3(1961), 1-47.

[5] M. Hedenqvist, U.W. Gedde, Diffusion of small molecule penetrants in semicrystalline polymers, Prog. Polym. Sci. , 21(1996), 299-333.

[6] G. Kirchhoff, Vorlesungen ueber de theorie der waerme, Barth, Leipzig (1894).

[7] G. Mittelman, J.H. Davidson, S.C. Mantell, Y. Su, Prediction of polymer tube life for solar hot water systems: A model of antioxidant loss, Solar Energy , 82 (2008), 452-461.

[8] P. Neogi, Diffusion in Polymers, Marcel Dekker, New York (1996).

[9] F. Pigeonneau, B. Jaffrennou, A. Leailleur K. Limouzin, Numerical investigation of a generalized Graetz problem in circular tube with a mass transfer coupling between the solid and the liquid, Int. J. Heat Mass Transf., 96 (2016), 381-395.

[10] O. Vitrac, M. Hayert, Risk assessment of migration from packaging materials into foodstuffs, A.I.Ch.E. J. , 51 (2005), 1080-1095.

[11] O. Vitrac, A. Mougharbel, A. Feigenbaum, Interfacial mass transport properties which control the migration of packaging constituents into foodstuffs, J. Food Engng. , 79 (2007), 1048-1064.

Gheorghe JUNCU,

POLITEHNICA University Bucharest,

Department of Chemical and Biochemical Engineering,

Polizu 1, 011061 Bucharest, România.

Email: juncu@cael.pub.ro 